\title{
Graphene Characterization using Time-Domain Terahertz Spectroscopy for Plasmonic Antenna Design
}

\author{
Farah Vandrevala \\ University at Buffalo \\ Buffalo, New York \\ farahper@buffalo.edu \\ Josep Miquel Jornet \\ University at Buffalo \\ Buffalo, New York \\ jmjornet@buffalo.edu
}

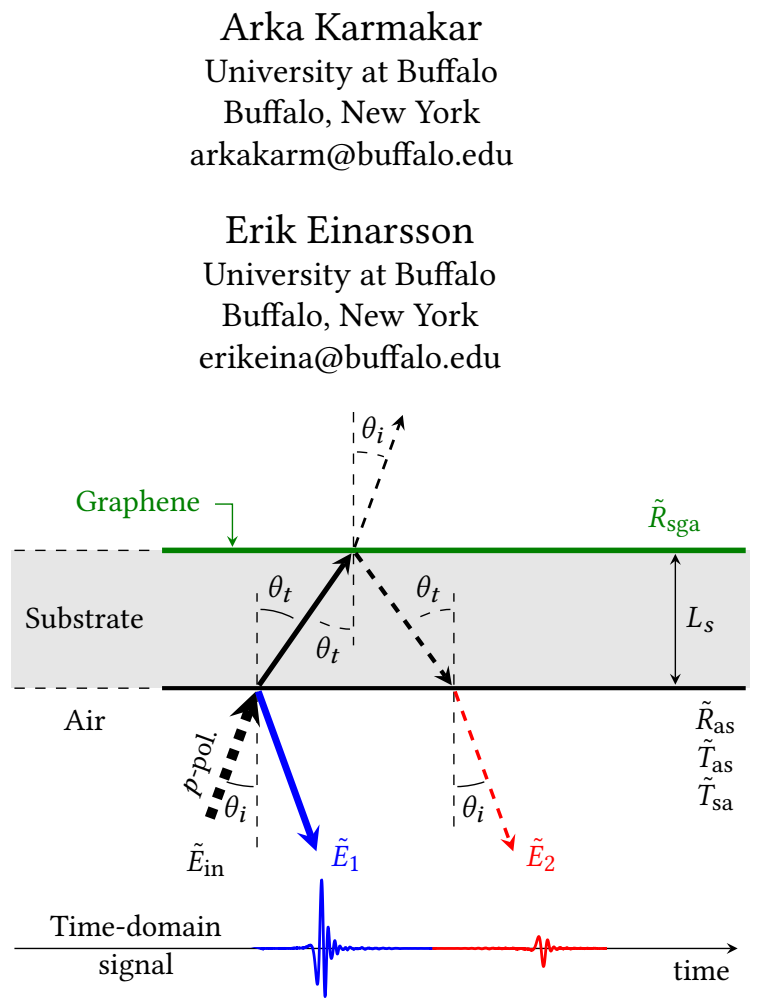

Figure 1: (Top) Ray diagram for $\mathrm{THz}$ light incident on the graphene-on-substrate system. (Bottom) Measured THz pulses corresponding to interface reflections.

network is expected to lead to an Internet of Nano-Things [1], which has the potential to revolutionize how we live and work.

Graphene boasts a long list of impressive physical properties [2]. Among these is the ability to support collective charge oscillations, known as surface plasmons, at a graphene-dielectric interface [3]. These surface plasmons can couple to electromagnetic (EM) waves, forming surface plasmon polaritons that strongly confine the freespace EM wave at the interface. Appropriate graphene dimensions would thus define a resonant cavity that should act as a plasmonic antenna in the $\mathrm{THz}$ range [5].

In this work, we use terahertz time-domain spectroscopy (THzTDS) to extract the complex optical properties of CVD-grown graphene transferred onto an undoped silicon substrate. We do this by analyzing the light-matter interactions induced by picosecond pulses of THz light at the substrate-graphene interface as shown in Fig. 1. We measure a time-domain signal consisting of two pulses corresponding to the reflection at each interface. This is shown at the bottom of Fig. 1. We use a Hann window function centered at the peak of each pulse to separate the signal into two parts (red/blue in Fig. 1). We can then define a transfer function, $\tilde{H}$, using the echo pulse as the output and the main pulse as the input. 
Wavelength, $\lambda(\mu \mathrm{m})$

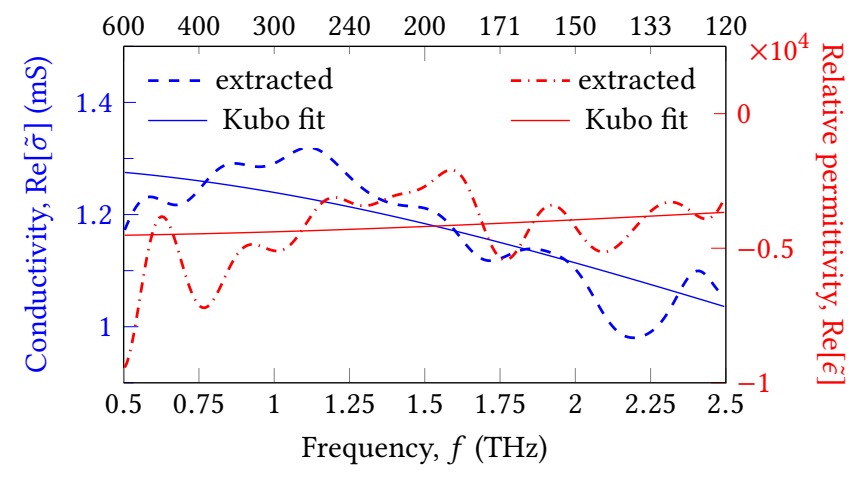

Figure 2: Extracted optical properties of graphene

\section{THZ ANALYSIS AND CHARACTERIZATION}

Our self-referenced measurement setup yields the reference substrate optical properties, $\tilde{n}_{\mathrm{s}}=n_{\mathrm{s}}-j \kappa_{\mathrm{s}}$, in a single step through the transfer function equation defined below:

$$
\tilde{H}=\frac{\left|\tilde{E}_{2}\right|}{\left|\tilde{E}_{1}\right|} \exp \left(-j\left[\phi_{2}-\phi_{1}\right]\right)
$$

Based on an approach similar to [4], we calculate the Fresnel reflection coefficient at the substrate-graphene-air interface as follows:

$$
\tilde{R}_{\text {sga }}=\tilde{H}_{\mathrm{g}} \frac{\tilde{R}_{\mathrm{as}}}{\tilde{T}_{\mathrm{as}} \tilde{T}_{\mathrm{sa}}} \exp \left(j \frac{\omega}{c} 2 \tilde{n}_{\mathrm{s}} L_{\mathrm{s}} \cos \theta_{t}\right)
$$

We then extract the complex conductivity [8] using the equation:

$$
\tilde{\sigma}=\frac{1}{Z_{0}}\left[\left(\frac{1-\tilde{R}_{\text {sga }}}{1+\tilde{R}_{\text {sga }}}\right) \frac{\tilde{n}_{\mathrm{s}}}{\cos \theta_{t}}-\frac{1}{\cos \theta_{i}}\right]
$$

Based on Maxwell's equations, the complex dielectric function is

$$
\tilde{\epsilon}=\epsilon_{0}+j \frac{\tilde{\sigma}}{\omega}=\epsilon_{0}-\frac{\operatorname{Im}[\tilde{\sigma}]}{\omega}+j \frac{\operatorname{Re}[\tilde{\sigma}]}{\omega}
$$

Since the real part of the dielectric function is related to the imaginary part of the complex conductivity, we fit our data, as shown in Fig. 2, using the Kubo formalism defined below, which is commonly used to model the intra-band surface conductivity of graphene [7].

$$
\tilde{\sigma}=\frac{\tau}{1-j \omega \tau}\left(\frac{2 e^{2}}{\pi \hbar^{2}} k_{B} T\left[2 \cosh \left(\frac{E_{F}}{2 k_{B} T}\right)\right]\right)
$$

Here the Fermi energy of graphene is given by

$$
E_{F}=\frac{\pi \hbar^{2}}{e^{2}} \frac{\sigma_{\mathrm{dc}}}{\tau}
$$

in which $\sigma_{\mathrm{dc}}$ is the dc conductivity and $\tau$ is the scattering time.

\section{RESULTS}

Based on the fitting to the Kubo formalism, we extracted the electrical parameters given in Table 1. We used the COMSOL Multiphysics platform to solve Eqs. (4) and (5) and simulate the electric field inside a graphene patch antenna. Figure 3a uses a calculated value of the scattering time [6], which shows the electric field of the surface plasmon being able to traverse the entire length of the patch. On
Table 1: Electrical parameters of graphene

\begin{tabular}{lcc}
\hline Parameter & Symbol & Value \\
\hline dc conductivity & $\sigma_{\mathrm{dc}}$ & $1.3 \mathrm{mS}$ \\
Scattering time & $\tau$ & $31.5 \mathrm{fs}$ \\
Fermi energy & $E_{\mathrm{F}}$ & $0.35 \mathrm{eV}$ \\
Carrier density & $N$ & $1.4 \times 10^{13} \mathrm{~cm}^{-2}$ \\
Carrier mobility & $\mu$ & $576 \mathrm{~cm}^{2} \mathrm{~V}^{-1} \mathrm{~s}^{-1}$ \\
\hline
\end{tabular}

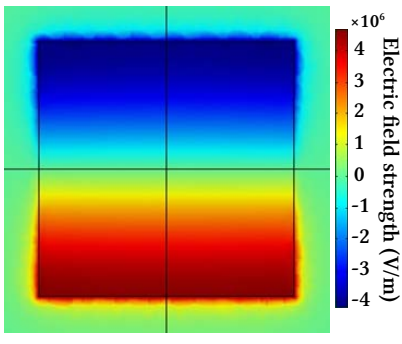

(a) Using ideal $\tau=0.5 \mathrm{ps}$

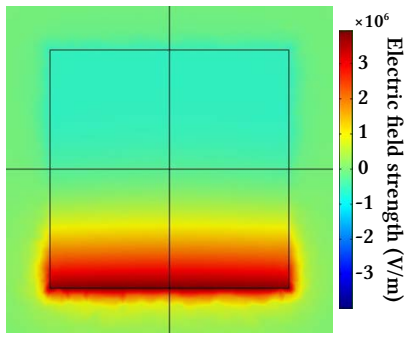

(b) Using extracted $\tau=31.45 \mathrm{fs}$
Figure 3: Electric field simulations for plasmon excitation in graphene patch antenna for different scattering times.

the other hand, Fig. 3b shows that if the extracted value of $\tau$ is used, then the electric field of the surface plasmon decays very quickly. A distinct advantage of THz-TDS over traditional electrical measurements is that, due to its non-contact nature, there is no risk of influencing or compromising the sample during measurement.

\section{CONCLUSION}

Here we report THz-TDS to be a robust optical and electrical characterization method for graphene, especially for applications involving plasmonic antennas. The clear difference seen in running simulations using predicted, or theoretical, values versus real-world values accentuates the importance of obtaining graphene parameters under real-world conditions to guide antenna design and better evaluate expected performance.

\section{REFERENCES}

[1] I. F. Akyildiz and J. M. Jornet. 2010. The Internet of Nano-Things. IEEE Wireless Communications Magazine 17, 6 (Dec. 2010), 58-63.

[2] A. K. Geim and K. S. Novoselov. 2007. The rise of graphene. Nature Materials 6, 3 (March 2007), 183-191.

[3] E. H. Hwang and S. Das Sarma. 2007. Dielectric function, screening, and plasmons in two-dimensional graphene. Physical Review B 75 (May 2007), 205418. Issue 20.

[4] Peter Uhd Jepsen, Uffe Møller, and Hannes Merbold. 2007. Investigation of aqueous alcohol and sugar solutions with reflection terahertz time-domain spectroscopy. Opt. Express 15, 22 (2007), 14717-14737.

[5] T Otsuji, S A Boubanga Tombet, A Satou, H Fukidome, M Suemitsu, E Sano, V Popov, M Ryzhii, and V Ryzhii. 2012. Graphene-based devices in terahertz science and technology. Fournal of Physics D: Applied Physics 45, 30 (2012), 303001.

[6] N. Sule, K. J. Willis, S. C. Hagness, and I. Knezevic. 2014. Terahertz-frequency electronic transport in graphene. Physical Review B 90, 4 (2014), 045431.

[7] Luke Zakrajsek, Erik Einarsson, Ngwe Thawdar, Michael Medley, and Josep Miquel Jornet. 2016. Lithographically Defined Plasmonic Graphene Antennas for Terahertz Band Communication. IEEE Antennas and Wireless Propagation Letters 15 (2016), 1553-1556.

[8] Yixuan Zhou, Yiwen E, Lipeng Zhu, Mei Qi, Xinlong Xu, Jintao Bai, Zhaoyu Ren, and Li Wang. 2016. Terahertz wave reflection impedance matching properties of graphene layers at oblique incidence. Carbon 96 (2016), 1129-1137. 